\title{
Proposal of a new real-time cooperative challenge in mobile robotics
}

\author{
P. Costa ${ }^{*, * *}$ A. Moreira ${ }^{*, * * *}$ J. Gonçalves ${ }^{* *, * * *}$ J. Lima Mi*,*** $^{* *}$ \\ * Faculty of Engineering of University of Porto, Department of \\ Electrical Engineering and Computers, Porto, Portugal, \\ e-mail: $\{$ paco,amoreira\}@fe.up.pt \\ ** Polytechnic Institute of Bragança, Department of Electrical \\ Engineering, Bragança, Portugal \\ e-mail: $\{$ goncalves,jllima $\}$ ipb.pt \\ *** Robotics and Intelligent Systems Research Group, INESC, Porto, \\ Portugal
}

\begin{abstract}
:
In this paper it is presented a new robot competition that is going to be included in Robotica 2011, the main Robotics Portuguese Competition. The robot competition takes place in an emulated factory plant, where Automatic Guided Vehicles (AGVs) must cooperate to perform tasks. To accomplish their goals the AGVs must deal with localization, navigation, scheduling and cooperation problems, that must be solved autonomously. One option of prototyping the AGVs is the use of the Lego Mindstorms NXT technology. The presented example can play an important role in education due to the inherent multi-disciplinary concepts that are involved, motivating students to technological areas. It also plays an important role in research and development, because it is expected that the outcomes that will emerge here, will later be transfered to other application areas, such as service robots and manufacturing.
\end{abstract}

Keywords: Robotics, Education, Manufacturing

\section{INTRODUCTION}

Robotic competitions are an excellent way to foster research and to attract students to technological areas compete. The robotic competitions present standard problems that can be used as a benchmark, in order to evaluate and to compare the performances of different approaches. Although there are many robotic competitions Lund and Pagliarinis [2000] Nakanishi et al. [2006] Browning et al. [2005] Ribeiro et al. [2004] Pagello et al. [2004], there is the need to create new ones, in order to solve new challenges. The factory environment is a prime candidate to use robots in a variety of tasks. A competition where mobile robots are tackling transportation problems in the shop floor is a challenge that can foster new advances in service robots and manufacturing Yuta et al. [2006]. This new robot competition presents problems that occur when using mobile robots to perform transportation tasks. The robots must be able to navigate, cooperate and to selflocalize in an emulated factory plant, to transport and handle materials in an efficient way.

The authors have previous experience in robotic competitions, they participated in all senior competitions of the main Robotics Portuguese Competition, as well as in several leagues in the Robocup. This participations included several roles, such as being part of technical committees, competing with teams and also as event organizers. The goals of each competition are defined having in mind the outcomes that are expected. The outcomes could be technical, in order to obtain better hardware and software performances, in research, promoting advances in some areas of knowledge and in education, depending on what students can learn and practice while they are involved in the competitions. As an example of a robot competition participation it is shown in Figure 1 the authors team (5DPO) at the Robocup 2006 Small Size League final, playing against Carnegie Mellon team (CMDragons).

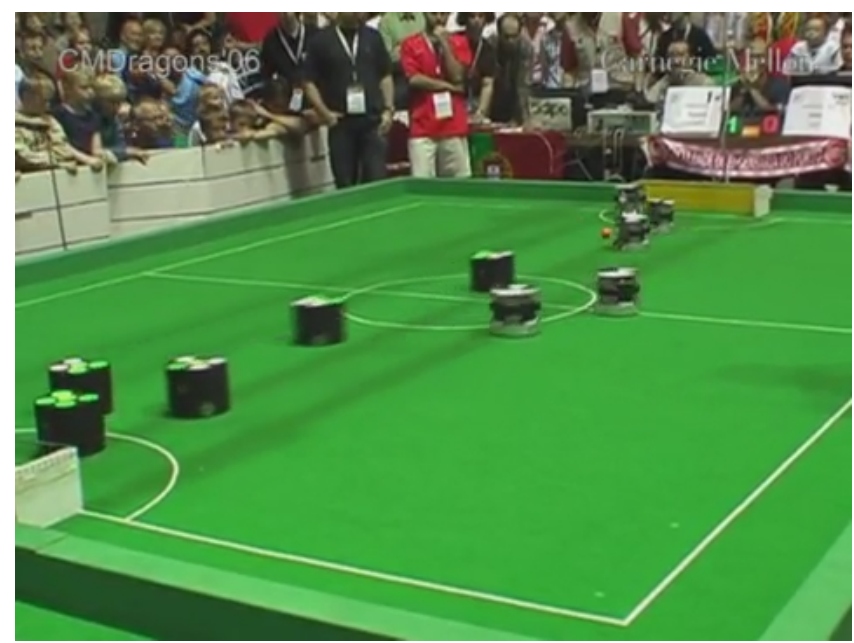

Fig. 1. 5DPO team in a Robocup Small Size League game

The paper is organized as follows: After a brief introduction the contest description where robots hardware, dimensions, materials and localization requisites and different 
difficulty levels are described. Then the official competition robot simulator is described, allowing competitors to test their approaches without accessing to real hardware. Then, an example of a particular case of the competition with Lego Mindstorms NXT is presented. Finally, some conclusions and future work are pointed out.

\section{ROBOTIC COMPETITION DESCRIPTION}

In this section it is presented the competition description and the rules that teams must follow in order to qualify for participation and to compete. This competition has already been accepted as an official competition of Robotica 2011, the Main Robotics Portuguese Competition, that is going to be held in Lisbon in April 2011, being expected to be motivating for robotics researchers and enthusiasts.

Although the competition requires real robots, competitors can use a simulator in order to test their approaches, before and during the competition. The official competition simulator is SimTwo, described in section 3, being a tool for the development and validation of robot software that can be freely downloaded Costa et al. [2010].

\subsection{Robot dimensions}

Each robot must fit within a cuboid of $35 \times 35 \times 30 \mathrm{~cm}$. The robot must be completely autonomous and cannot establish any kind of communication with an external system that is not explicitly provided by the organization.

\subsection{Competition arena}

The competition arena, shown in Figure 2, emulates a factory shop floor where there are warehouses and machinery. A real robot in the competition arena is also shown in Figure 3. The dimensions of this area is $3.5 \times 2.5 \mathrm{~m}$. There are eight machines available and two warehouses. One of them is used as a raw material storage and the other one is used as a destination.

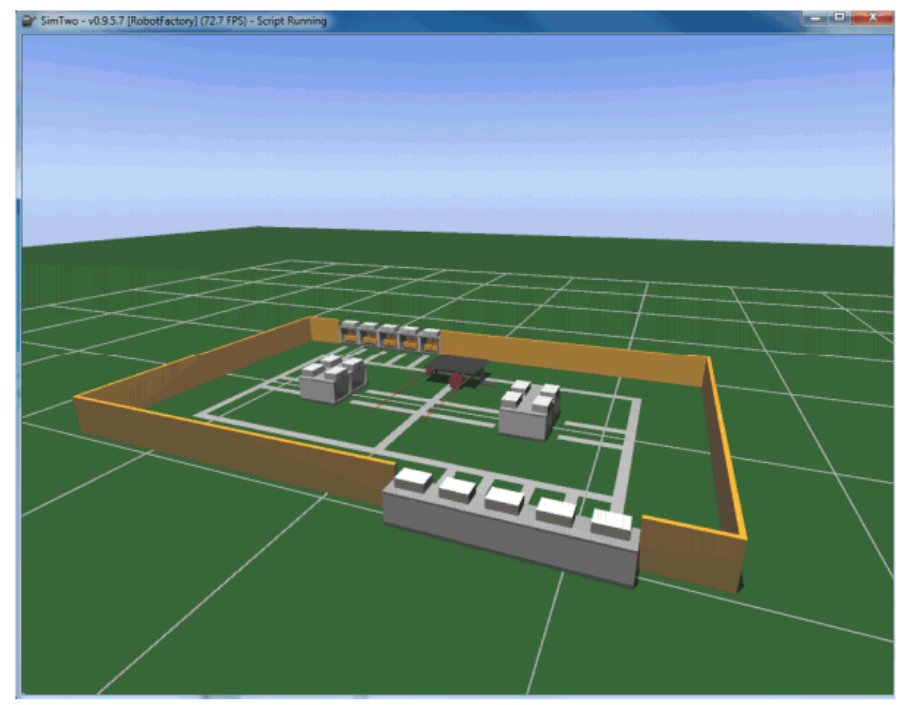

Fig. 2. Competition arena modeled in the SimTwo.

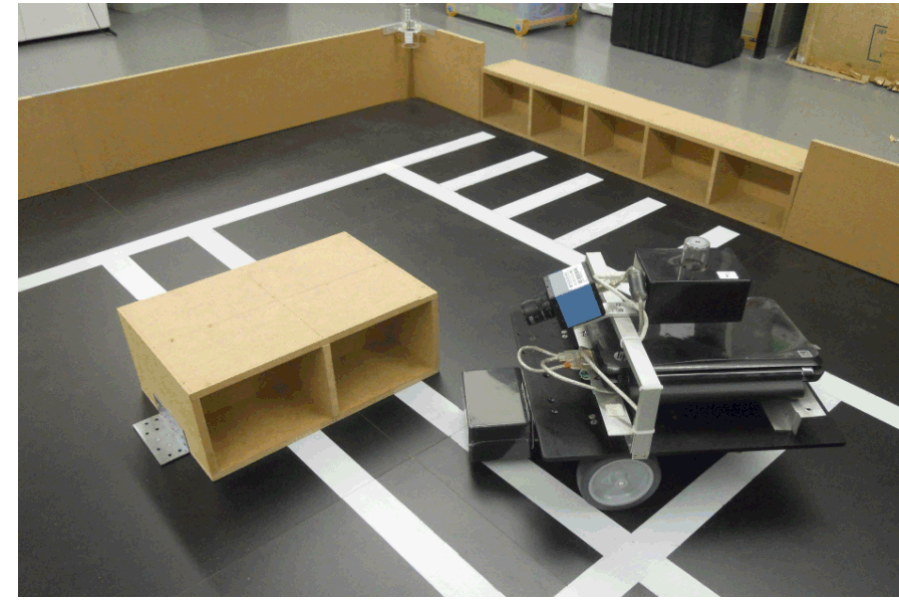

Fig. 3. Robot in the competition arena.

Machinery and warehouses description Each machine provides an area where the pieces should be placed in order to be processed by the machine. The robot must pick and place the material parts from the machine. While the part is placed in the machine it is processed and should not be removed. An RGB LED indicates that the machine is able to accept parts (light green), the machine is processing a part (yellow light), the part in this machine is already processed (white light) or that the machine is broken (blink red light).

The part materials The materials to be transported by the robots should respect standard dimensions, width and length corresponding to an Europallete 80 x $120 \mathrm{~mm}$ (1:10 scale), the height should have a value between $30 \mathrm{~mm}$ and $50 \mathrm{~mm}$. Each piece has an LED issuing an RGB color that identifies the type of material. When a part arrives to a machine, it can be processed and its color is changed in order to illustrate a different type of part.

\subsection{Solving problems in the competition}

Team responsible can access the robot up to four times, if one of the robots is not expected to be able to recover. While robot comes out from the arena the time scheduling continues unchangeable.

\subsection{Competition starting}

The robots must be placed in the park closed one hour before the start of each competition. Teams should not to have access to the robot until about 10 minutes before the start of their competition. There, the referees indicate the teams that should prepare the robot to start their competition.

\subsection{Competition rounds}

Since this is a competition that can accept participants with different background, it must be differentiated in three rounds. Event organization can provide, for some rounds, an external localization system for robots Moreira et al. [2001]. This system will identify the robots using a pattern that must be placed on top of each robot and can provide the position and orientation of the robot. 
First round The main purpose of the first round is to collect the pieces of the raw material warehouse and transport them to the end warehouse. The robot should transport the most parts it can from the warehouses.

Second round The main purpose of the second round is to process some parts of the raw material. The raw material should be transported from the initial warehouse to the machinery, in order to be processed. When the processing task is ended, the parts should be transported to the final warehouse.

Third round The main purpose of the third round is to sequentially distribute the parts through several machinery. Some parts collected from the raw material warehouse should be placed sequentially in more than one machine to process. Only after the completion of this operation the parts should be transported to the final warehouse. There will be three types of parts in operation. During this round some tracks may be partially or totally blocked. In this round teams are authorized to use two robot at the same time, the used robots must cooperate to perform its tasks.

\subsection{Expected education and research outcomes}

One educational aspect, other than the natural motivating factor, is the availability of a challenge that is harder than some entry level competitions but still reachable for teams with some experience on those tasks. Also, it is expected that the problem scales well to more complex approaches. This means that more complex robot, thus a harder robot to build, will also be able to perform better. This can be a excellent motivation for teams to learn and use more advanced robots and algorithms.

By presenting a scaled down factory shop, this competition creates a benchmark that can be used to compare different approaches to the problems that arise on this kid of environments. Also, the ability, in some restricted areas, alter the environment, can promote the test and evaluation of different localization mechanisms. Something that is usually, more restricted in most other competitions. That opens this area to be explored and benchmarked.

\section{SIMTWO - THE COMPETITION OFFICIAL SIMULATOR}

SimTwo is a realistic simulation system that can support several types of robots. Its main purpose is the simulation of mobile robots that can have wheels or legs, although industrial robots, conveyor belts and lighter-than-air vehicles can also be defined. Basically any type of terrestrial robot definable with rotative joints and/or wheels can be simulated in this software. Figure 4 shows the software with all its main windows.

The dynamics realism in SimTwo is obtained by decomposing a robot to rigid bodies and electric motors. Each body behaviour is numerically simulated using its physical characteristics: shape, mass and moments of inertia, surface friction and elasticity. It is also possible to define standard joints such as universal, hinge and slider which can be coupled with an actuator or a sensor.

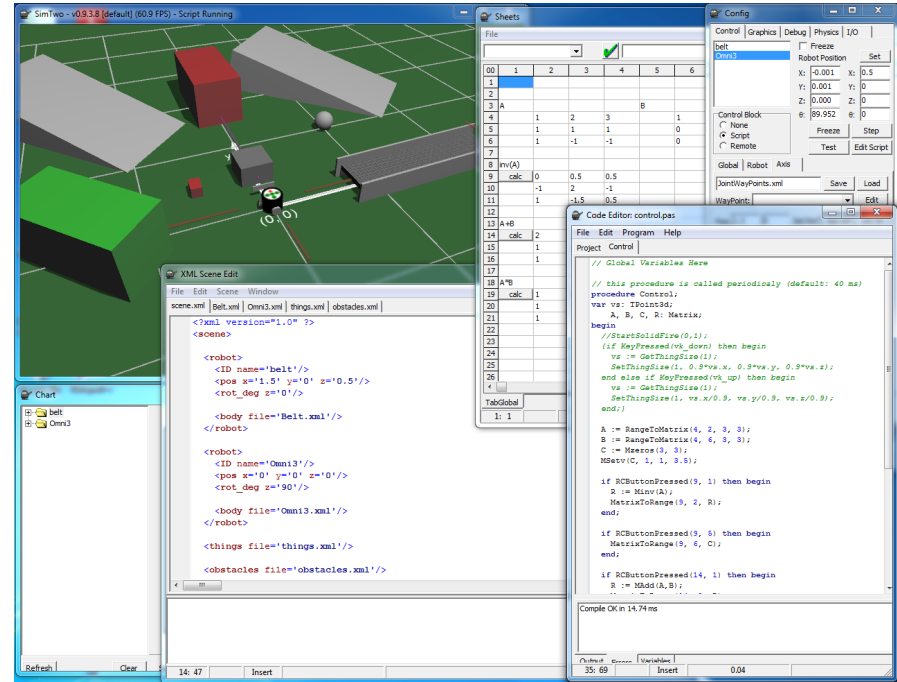

Fig. 4. SimTwo software. Application windows clockwise from top left corner: world view, spreadsheet, configuration, code editor, scene editor and chart.

SimTwo is an application with a multiple document interface (MDI) where all windows are under the "world view" window control, shown in Figure 4, exiting the simulator is done by closing this window. The "configuration" window offers control over several elements of the virtual scene. It is possible to define the controller timestamp and to configure the 3D world view (camera position, shadow visibility, etc.). Robots information is also displayed in this window, like its position and speed.

The "code editor" offers an integrated development environment (IDE) for high-level programming based in Pascal language, this is the main tool in this simulator. The control algorithms are directly compiled in this window and a message appears in the bottom of the page informing of a successful compilation or the existence of coding errors. The control script is started from this window where the resulting robot movement is visible in the main window and any changes to the control script requires this to be stopped and recompiled.

Debugging the control algorithm is possible using the "chart" and the "spreadsheet" window. In the "chart" it is possible to plot all variables available for every robot, such as its position, motors speed and current, etc. In the "spreadsheet" window it is possible to define "edit cells" as well as "button cells" for specific operations. This window becomes a customizable form window, this is the equivalent to a graphic application.

The scene implementation is done by editing several XML format files, these files are definable in the "scene editor" window. A scene in SimTwo can have "robots", "obstacles" and "things", as shown in Figure 5.

A main scene file (scene.xml) defines the robots in use and their specific construction file. Each robot is defined by various solids (cuboid, cylinder and sphere) connected through joints (slider, socket and hinge). The shell elements are solids without mass, these do not modified the robot physical properties but are an essential part for collision simulation. A robot can also have sensors, these 


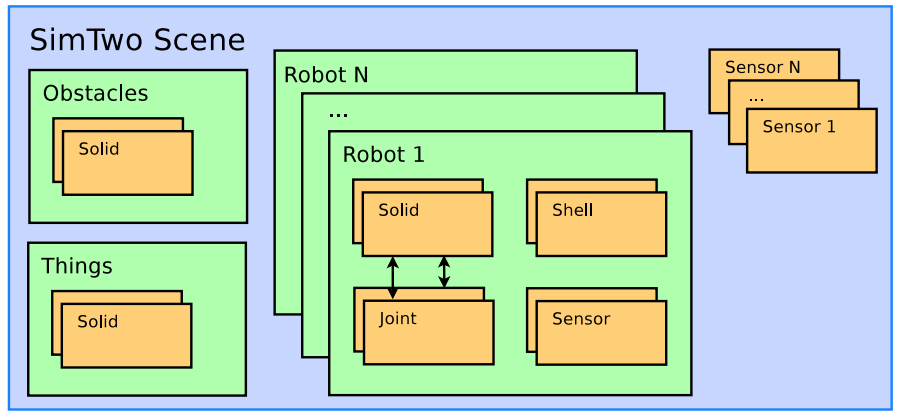

Fig. 5. SimTwo Scene structure

provide information from the environment surrounding the robot.

The scene objects are the "obstacles" and "things", these are very similar in definition (both are defined solely by solids) but while the "obstacles" are imovable in case of collision the "things" are not. If a robot collides with a "thing" these will react accordingly to their mass definition. A scene can also have sensors, these are static relative to the world as opposed to a robot sensor which gives information relative to its corresponding robot.

In Figure 6 it is presented the flux of information of a SimTwo controller. The controller has different levels that are updated at different rates, being presented its default values. The presented default values can be changed by the user depending on the application that is being simulated. The artificial intelligence (AI) controller is updated by default at $40 \mathrm{~ms}$, being a common rate to accomplish real time requisites in mobile robotics challenges. The motor controller is updated by default at a $10 \mathrm{~ms}$ rate and its model output is updated by default at $1 \mathrm{~ms}$ rate. The default values are typical values that were chosen having in mind the dynamics of the world and motor models in mobile robotics, although user can change this values depending on the specific dynamics and real time requisites of its simulation.

The artificial intelligence level is provided with sensor information extracted from the world state. After some decisions and calculations related with control, localization and navigation the controller provides to the motor controller its inputs. The motor model provides the torque information, forcing the Rigid Dynamics Engine to react and consequently forcing the World state (Sensor, obstacles and robot positions) to change.

\section{COMPETING WITH LEGO MINDSTORMS NXT}

It is very important that the Lego Mindstorms based robots can enter in the contest, having in mind this technology advantages in the rapid prototyping. That was an important requirement to promote the participation of those who are taking their first steps in the world of mobile robotics. The proposed competition is not restricted to the Lego Mindstorms use, being this technology one of the possibilities to prototype a robot in order to participate. The Lego modularity makes the rapid prototyping of different robot configurations easier. The Lego parts allow connectivity, suppressing the need of using glue or screws. It's a modular tool, it is possible for the same part to be a different thing depending on the application, motivating

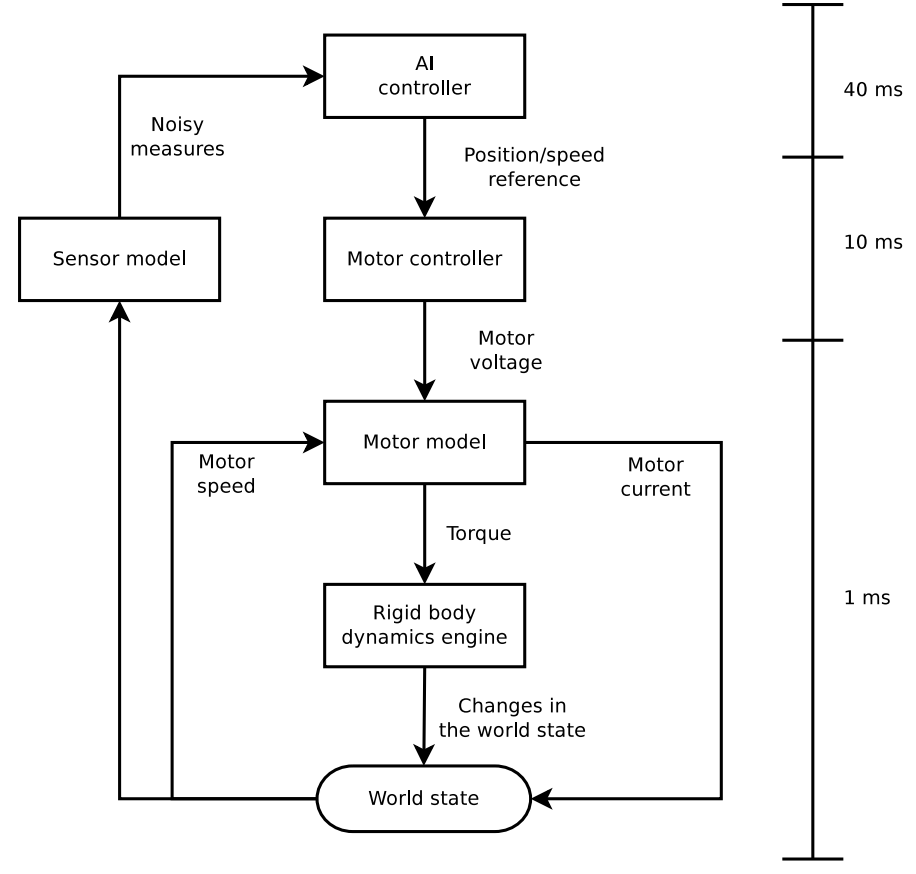

Fig. 6. SimTwo controller cycle

those who are using it and it is presented at a relatively low cost Gawthrop and McGookin [2006] Gonçalves et al. [2006].

\subsection{Lego Mindstorms NXT simulation}

The Lego Mindstorms can play an important role in the first years of competition because competitors can easily prototype a robot that fulfills all the requisites in order to compete and also to promote the participation of those who are taking their first steps in the world of mobile robotics. Added to all the advantages of the Kit Lego Mindstorms, simulation can also be a very important aid, allowing competitors to test their approaches without accessing to real hardware. An example of a simulated robot prototyped with Lego Mindstorms Technology is shown in Figure 7. The modeling and simulation of the Lego Mindstorms NXT was previously presented Gonçalves et al. [2009].

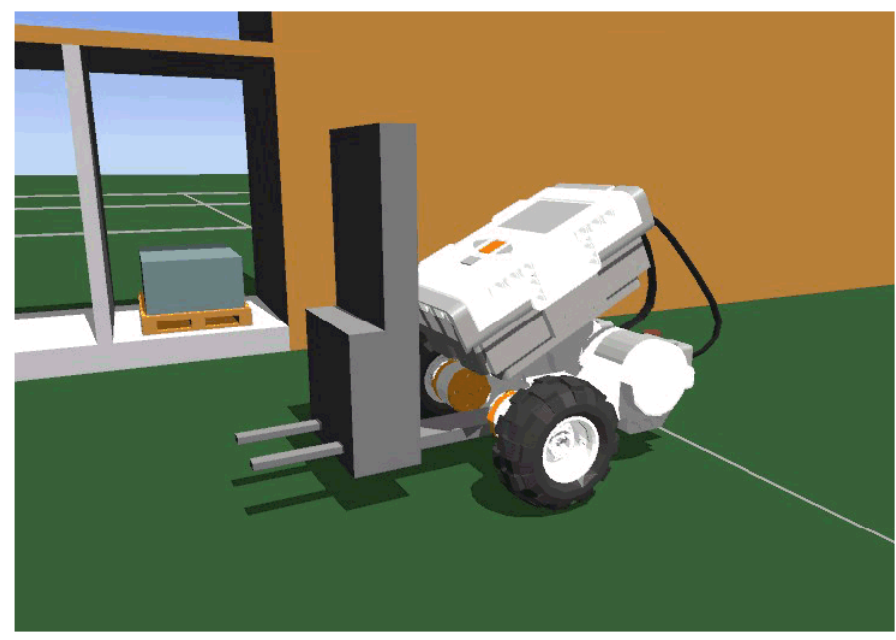

Fig. 7. Simulated Lego Mindstorms NXT in the arena. 
The simulated robot is provided, in the simulator, with its position in the arena. If the competitor is working with real hardware its position can be given by an external external localization system for robots Moreira et al. [2001], provided by the organization. This system will identify the robots using a pattern that must be placed on top of each robot and can provide the position and orientation of the robot. Team will be responsible to use the coordinates of the pattern to find the position and orientation of the robot, compensating the inevitable parallax error Tian et al. [2003]. The robots position information is provided via Bluetooth to the robots, so teams do not have to deal with localization problems, being only necessary for competitors to optimize the trajectories so that robots can perform its tasks as fast as possible. The competing teams are welcome to develop their own localization systems.

\subsection{Lego Mindstorms prototype}

An example of a robot prototyped for the robot competition is shown in Figure 8.

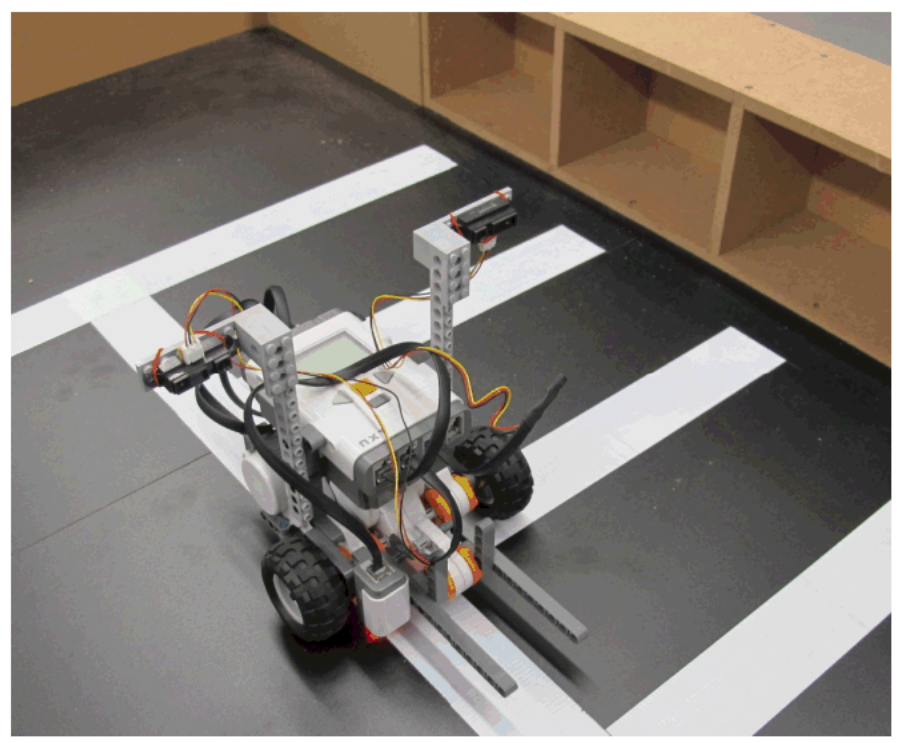

Fig. 8. Lego Mindstorms NXT in the arena.

The robot was prototyped almost exclusively with Lego Mindstorms components with the exception of the used distance sensor. The used distance sensor is a Sharp infrared distance sensor, being easily interfaced to an NXT input Port and providing a good performance for small distances. A brief description of the infra-red distance sensor is given in the subsection 4.2.1.

The presented robot does not use the localization system provided by the organization, in order to navigate the robot reads reflected light from the floor resorting to a light sensor. The amount of the reflected light is different if the robot is standing on top a white line. The robot to navigate also uses the distance sensor measures and odometry calculation. The distance sensor measures can be used when the robot is approaching a warehouse or a machine, providing data to calculate the robot localization relatively to the environment that surrounds him. The odometry calculation provides very good results in a short term being possible to fuse this data with the distance sensors absolute measurements resorting to an extended Kalman filter Gonçalves et al. [2008] Thrun et al. [2005] Choset et al. [2004].

Sharp infrared distance sensor The Sharp family of infrared range finders is very popular for projects that re-quire cheap and somewhat accurate distance measurements. Some drawback of these sensors is their non-linear response and its mandatory minimum distance measurement requisites. Their inherently fast response is attractive for enhancing the real-time response of a mobile robot Benet et al. [2002]. Some IR sensors are based on the measurement of the phase shift, and offer medium resolution from $5 \mathrm{~cm}$ to $10 \mathrm{~m}$ Mohammad [2009], but these are very expensive.

This IR sensor is more economical than sonar rangefinders, yet it provides much better performance than other IR alternatives. Interfacing to most micro-controllers is straightforward: the single analog output can be connected to an analog-to-digital converter for taking distance measurements, or the output can be connected to a comparator for threshold detection. The detection range, suggested by the manufacturer Sharp [2010], of this version is approximately $10 \mathrm{~cm}$ to $80 \mathrm{~cm}$, but it offers other models with different measuring ranges. The tested IR sensor (GP2Y0A21YK0F) uses a 3-pin JST connector, when looking at the back, the three connections from left to right are power, ground, and the output signal, as shown in Figure 9.

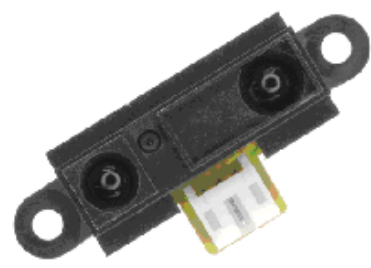

Fig. 9. Sharp infrared sensor.

A low-pass filter in the sensor output allows a cleaner measure, having a time constant of $1 \mathrm{~ms}$ which is considerably lower than the sensors response time of $38 \mathrm{~ms}$. In Figure 10 is shown a representation of the used electronic circuit.

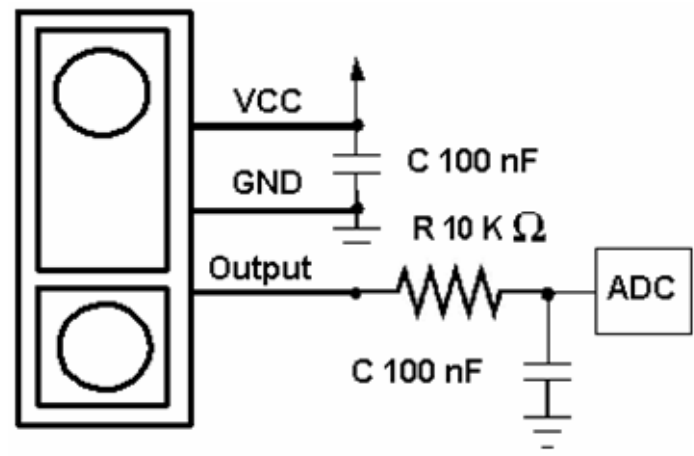

Fig. 10. Sensor electronic conditioning circuit. 
According to the information provided by the manufacturer in a sensor application note Sharp [2010], the voltage inverse can be approximated to a straight line in the working range. Figure 11 shows the inverse voltage in function its distance with the respective linear regression.

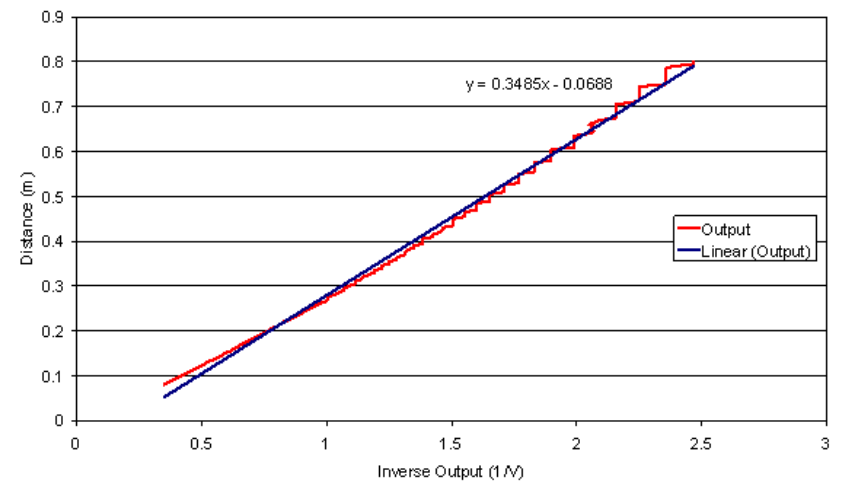

Fig. 11. Distance to inverse voltage output curve and linear regression

\section{CONCLUSIONS AND FUTURE WORK}

In this paper it is presented a new robot competition that is going to be included in the main Robotics Portuguese Competition. The robot competition takes place in an emulated factory plant, where Automatic Guided Vehicles (AGVs) must cooperate to perform tasks. To accomplish its goals the AGVs must deal with localization, navigation, scheduling and cooperation problems, that must solved autonomously.

One of the options to prototype the AGVs is the use of the Lego Mindstorms NXT. The purpose of the use of this technology is to facilitate competitors to fulfill competition requisites, having in mind the Lego Mindstorms advantages in rapid prototyping. It is also very important to promote the participation of persons who are taking their first steps in the world of mobile robotics.

While competitors do not have contact with physical arena and hardware, competitors can freely download the official competition simulator to test their controllers.

The first official competition will be held in Lisbon at Robotica 2011, the Main Robotics Competition. The authors expect to receive feedback from the first participants in order to evolve the rules of the competition to a more mature state, having in mind what students can learn and practice while they are involved in the competition and also to obtain new technical and research advances. It it is expected that the research and technical outcomes that will emerge here, will later be transfered to other application areas, such as service robots and manufacturing.

\section{REFERENCES}

Benet, G., Blanes, F., Simo, J., and Perez, P. (2002). Using infrared sensors for distance measurement in mobile robots. In Journal of Robotics and Autonomous Systems. vol. 10,pp. 255-266.

Browning, B., Bruce, J., Bowling, M., and Veloso, M. (2005). Ustp: Skills, tactics and plays for multi-robot control in adversarial environments. IEEE Journal of Control and Systems Engineering.

Choset, H., Lynch, K., Burgard, W., Kavraki, L., and Thrun, S. (2004). Principles of robot motion. MIT Press. Costa, P., Gonçalves, J., Lima, J., and Malheiros, P. (2010). Simtwo realistic simulator: A tool for the development and validation of robot software. Accepted for publication in the International Journal of Theory and Applications of Mathematics Computer Science.

Gawthrop, P. and McGookin, E. (2006). Using lego in control education. in Proceedings of the 7th Ifac Symposium on Advances in control education.

Gonçalves, J., Lima, J., and Costa, P. (2006). Rapid prototyping of mobile robots extending lego mindstorms platform. in Proceedings of the 7th Ifac Symposium on Advances in control education.

Gonçalves, J., Lima, J., Malheiros, P., and Costa, P. (2009). Realistic simulation of a lego mindstorms nxt based robot. Invited CCA Session "LEGO based Control Education and Prototyping in Robotics, Mechatronics and Embedded Systems, IEEE Multi-conference on Systems and Control", Saint Petersburg, Russia,.

Gonçalves, J., Lima, J., Oliveira, H., and Costa, P. (2008). Sensor and actuator modeling of an realistic wheeled mobile robot simulator. Proceedings of the 13th IEEE International Conference on Emerging Technologies and Factory Automation.

Lund, H. and Pagliarinis, L. (2000). Robocup jr. with lego mindstorms. in Proceedings of the 2000 IEEE International Conference on Robotics and Automation, San Francisco, CA, IEEE.

Mohammad, T. (2009). Using ultrasonic and infrared sensors for distance measurement. In World Academy Of Science, Engineering And Technology. Volume 51.

Moreira, A., Sousa, A., and Costa, P. (2001). Vision based real-time localization of multiple mobile robots. In 3rd Int. Conf. on Field and Service Robotics, Helsinki, Finland.

Nakanishi, R., Bruce, J., Murakami, K., Naruse, T., and Veloso, M. (2006). Cooperative 3-robot passing and shooting in the robocup small size league. In Proceedings of the RoboCup Symposium. Bremen, Germany.

Pagello, E., Menegatti, E., Bredenfeld, A., Costa, P., Christaller, T., Jacoff, A., Polani, D., Riedmiller, M., Saffiotti, A., Sklar, E., and Tomoichi, T. (2004). Robotic competition based education in engineering. AI Magazine, 25(2).

Ribeiro, F., Moutinho, I., Silva, P., Fraga, C., and Pereira, N. (2004). Controlling omni-directional wheels of a robocup msl autonomous mobile robot. In Scientific Meeting of the Portuguese Robotics Open.

Sharp (2010). Gp2y0a21yk0f sensor. In Datasheet.

Thrun, S., Burgard, W., and Fox, D. (2005). Probabilistic robotics. MIT Press.

Tian, Z., Kyte, M., and C.Messer (2003). Parallax error in video-image systems. Journal of Transportation Engineering, 128(3), 218-223.

Yuta, S., Asama, H., Thrun, S., Prassler, E., and Tsubouchi, T. (eds.) (2006). Field and Service Robotics, Recent Advances in research and Applications, FSR 2003, Lake Yamanaka, Japan, 14-16 July 2003, volume 24 of Springer Tracts in Advanced Robotics. Springer. 